\title{
Scaling Laws for Microemulsions Governed by Spontaneous Curvature
}

\author{
T. Tlusty, ${ }^{1}$ S. A. Safran, ${ }^{1}$ R. Menes, ${ }^{1}$ and R. Strey ${ }^{2}$ \\ ${ }^{1}$ Department of Materials and Interfaces, The Weizmann Institute of Science, Rehovot 76100, Israel \\ ${ }^{2}$ Max-Planck-Institut für Biophysikalische Chemie, Postfach 2841, 37018 Göttingen, Germany
}

(Received 30 September 1996)

\begin{abstract}
We introduce a model for microemulsions whose basic building blocks are cylindrical tubes connected by spherical junctions forming a network. The model predicts analytic scaling laws which quantitatively reproduce several prominent experimental features of the phase diagram, including the closed loops of 2-phase coexistence and the 3-phase body. The interfacial nature of our model, which takes into account only the curvature energy and the entropy of the interface, explains the observed water/oil symmetry and the collapse of the experimental data onto a single universal scaling curve. [S0031-9007(97)02840-8]
\end{abstract}

PACS numbers: $82.70 . \mathrm{Kj}, 64.70 . J a, 64.75 .+\mathrm{g}$

Recent studies of nonionic microemulsions (ME) have provided systematic experimental knowledge concerning their structure and phase behavior. The phase diagrams of these simplest ternary systems, which contain water, oil, and a nonionic surfactant, are fairly universal. Among the prominent features is the coexistence of either two or three phases in a temperature regime around $\bar{T}$, the temperature where the average curvature of the surfactant interfacial film vanishes [1]. The progression of the multiphase regions as a function of temperature exhibits a remarkable water/oil symmetry: As the temperature is increased above a critical value, $T_{-}<\bar{T}$ a two-phase closed loop with two critical points appears in the water-rich corner of the phase diagram (the oil volume fraction $\phi \sim 0.1$ ). A symmetrical loop appears in the oil-rich corner when the temperature is decreased below the critical value $T_{+}>\bar{T}$ [2]. When the temperature further approaches $\bar{T}$ (increasing in the water side and decreasing in the oil side) the loops start to expand. At certain temperatures the loops ( $T_{l}>T_{-}$on the water side, $T_{u}<T_{+}$on the oil side) intersect the emulsification failure line (EF) associated with the coexistence of globules and an excess phase [3]. This results in the creation of the three-phase body which exists in the temperature regime $T_{l}<T<T_{u}$ [4].

The microstructure of ME has been studied using NMR [5], neutron and x-ray scattering [6], and freeze fracture electron microscopy (FFEM) [7]. The experiments suggest that the ME near the closed loops and three-phase regions is locally cylindrical. However, the global geometry of the ME has not yet been established. There is some evidence (FFEM, x-ray scattering [8], viscosity, and conductivity experiments) that supports the existence of a connected structure [9].

Much effort has been directed at developing a theoretical understanding of ME which can explain in a unified manner both the phase equilibria patterns and microstructure observed in experiments [10]. The random interface models $[10,11]$ which describe the sponge phase do not reproduce the typical phase behavior of the closed loops and the critical points near the asymmetrical three-phase body, where the microstructure of the ME is asymmetric bicontinuous or globular. The important length scale in these asymmetric phases, far from $\bar{T}$, is the radius of curvature while the natural length scale in these models is the persistence length arising from the random collisions of fluctuating sheets.

In another class of ME models which treat more dilute ME phases composed of compact objects such as spherical droplets [3] or cylinders [12] the basic length scale is the spontaneous curvature. This approach was used in the work of Menes et al. [13] in order to explain the closed loops in terms of an interplay between a shape transformation and a postulated attraction between globules. In order to reproduce the observed closed loops the model had to assume that these short-ranged, material-specific interactions were in a particular window of attraction strengths. The loops obtained from this model were not as symmetrical as those observed in experiments [9]; moreover the shape dependent attraction mechanism required the assumption that the interactions of water globules in oil and oil globules in water be of the same order of magnitude.

Motivated by the experimental evidence we propose a theory based on a picture of branched tubular ME. The connected topology enables a unified description of both dilute systems in the vicinity of the loops, and the bicontinuous dense sponge $(\phi \sim 0.5)$. By modifying the compositions, the radius of the cylindrical tubes changes and the system continuously transforms from a dilute network composed of long narrow cylinders connected by remote junctions to a very dense network in which the radius of the cylinders is comparable with their length and the junctions are very close, which can very well represent the asymmetric bicontinuous sponge phase [8]. We show that the interplay between entropy and curvature energy which are coupled through the connected topology of the network can produce an effective attraction between the junctions. This, inherent, material independent entropic interaction is balanced by the steric repulsion of the undulating cylinders. We show that the observed phase 
behavior that stems from the detailed balance of these curvature-entropy interactions reproduces quantitatively many experimentally observed features of the closed loops and three-phase body. The water-oil symmetry is a generic feature of this model due to the entropic and interfacial character of the interactions.

We treat the branched ME as a network of selfassembling, semiflexible polymers $[12,13]$ interconnected by $z$-fold junctions [14]. The curvature energy associated with the creation of each junction, $\epsilon(r)$, to be calculated below, is a function of the cylinder radius, $r \sim \delta \phi / \phi_{s}$, where $\phi$ and $\phi_{s}$ are the volume fractions of the internal phase and surfactant, respectively, and $\delta$ the surfactant chain length. The junctions connect an ensemble of cylindrical branches whose length distribution is proportional to $X(m)$, the number density of branches of length $m$. The free energy per unit volume of the self-assembling network, $F_{N}$ (in units of $k_{B} T$ ), includes three contributions,

$$
F_{N}=\int X(m) \ln X(m) d m-(z-1) \rho \ln \rho+\rho \epsilon,
$$

where $\rho=\frac{2}{z} \int X(m) d m$ is the number density of the junctions. The first term is the Flory-type translational entropy of the free cylinders, the second term accounts for the entropy loss of the cylinders' free ends when they are constrained to meet at a junction, and the third term is the curvature energy of the junctions, that also includes the curvature energy of the cylinders which is independent of $X(m)$ [15].

The end-cap energy of a free cylinder is generally large compared to that of a junction, hence the system prefers to pay the entropy penalty of a junction in order to avoid free ends [16]. The optimal geometry of the network is found by minimizing $F_{N}$ with respect to $X(m)$. This procedure yields an exponential length distribution of the cylinders with an average cylinder length, $\bar{m}$, which scales like $\bar{m} \sim \phi^{1-z / 2} e^{\epsilon}$. Substituting the optimal distribution $X(m)$ results in

$$
F_{N}=-\rho=\frac{2}{z} \frac{\phi}{\bar{m}} \sim-\phi^{z / 2} e^{-\epsilon} .
$$

This is just the free energy of an ideal gas of junctions, whose density, $\rho$, is determined by the network volume fraction and the Boltzmann factor of the junction energy, $e^{-\epsilon}$. The $\phi^{z / 2}$ dependence of $F_{N}$ indicates that high- $z$ junctions are less probable [14]. We therefore consider only the case $z=3$. The nonlinear $\phi^{3 / 2}$ dependence of $F_{N}$ represents an effective attraction between the junctions.

As we show below, we find that the curvature energy of a junction, $\epsilon\left(c_{0} r\right)$ is a nonmonotonic function of the tube radius, $r$ and the spontaneous radius of curvature, $c_{0}^{-1}$. This nonmonotonic behavior is responsible for the trend of phase separation followed by remixing which produces the closed loops and three-phase body in the phase diagram.
We use a variational model to simplify the calculation of the curvature energy of the junction characterized by a bending modulus $\kappa(T)$. We consider a junction composed of three "horns" connected to a spherical core. The radius, $r$, of the cylindrical part of the horns is determined by the composition, and the parameter to be minimized is the curvature of the horn near the spherical core [17]. The minimized junction energy exhibits a nonmonotonic dependence on the tube radius, which has the approximate form [18],

$$
\epsilon(r, T) \simeq \frac{\kappa(T)}{T} \epsilon_{0}\left[1+\epsilon_{2}\left(c_{0} r-\frac{1}{2}\right)^{2}\right],
$$

where $\epsilon_{0} \simeq 2 ; \epsilon_{2} \simeq 10$, thus there is a deep minimum at $c_{0} r=1 / 2$ ( $r$ is measured in units in which the EF is at $\left.c_{0} r=1\right)$. As a result, Eq. (2) indicates that the effective attraction between the junctions, whose magnitude is proportional to $e^{-\epsilon}$, exhibits a steep maximum at $c_{0} r=$ $1 / 2$. Hence, increasing $r$ from the lower side of this maximum increases the effective attraction eventually driving the system to phase separate into two phases. A further increase in the radius decreases the attraction so that entropy tends to remix the phases. As shown below this phenomenon results in the creation of closed loops.

The effective attraction, $F_{N}=-\rho$, competes with two repulsive terms: One term arises from the restriction of the thermal fluctuations of the flexible tubes by neighboring cylinders resulting in an entropy decrease and an effective repulsion. Scaling arguments show that this repulsion behaves like $F_{H}=\frac{27}{16} \phi^{4 / 3}$, where the geometrical prefactor is found by a more detailed calculation [19]. The second term is a quadratic excluded-volume steric repulsion, $(1-\phi) \ln (1-\phi) \sim \phi^{2} / 2$. The dependence on the tube radius, $r \sim \delta \phi / \phi_{s}$, enters only in the attraction term, through the Boltzmann factor, $e^{-\epsilon(r, T)}$. The total free energy possesses a critical point where the attraction $F_{N}$ is large enough for the ME to phase separate. The phase of lower volume fraction is a dilute network with fewer junctions and the higher volume fraction is a dense network with many junctions [20]. The critical composition and junction energy are $\phi_{c} \simeq 1 / 8 ; e^{-\epsilon_{c}} \simeq$ 0.2 , respectively. The location of the critical point, which is far from the binary-mixture sides of the phase triangle, indicates that the system phase behavior is governed by the swollen network interactions and is not directly connected to the micellar binary systems. The nonmonotonic behavior of $\epsilon(r)$ indicates that there are two critical radii, $r_{c}$, at which the system phase separates. This provides an inherent mechanism for reentrant phase separation [13] which does not involve any material-specific interactions. The corresponding phase diagram is the closed loop with two critical points (Fig. 1).

The temperature dependence enters exponentially through the prefactor $\kappa(T) / T$ of the Boltzmann factor argument of the attraction. $\kappa(T)$ is the renormalized bending modulus [21]: $\quad \kappa(T) \simeq \kappa_{0}[1-(3 / 4 \pi)(T /$ $\left.\left.\kappa_{0}\right) \ln (r / a)\right]$. When we approach $\bar{T}$, the temperature at 


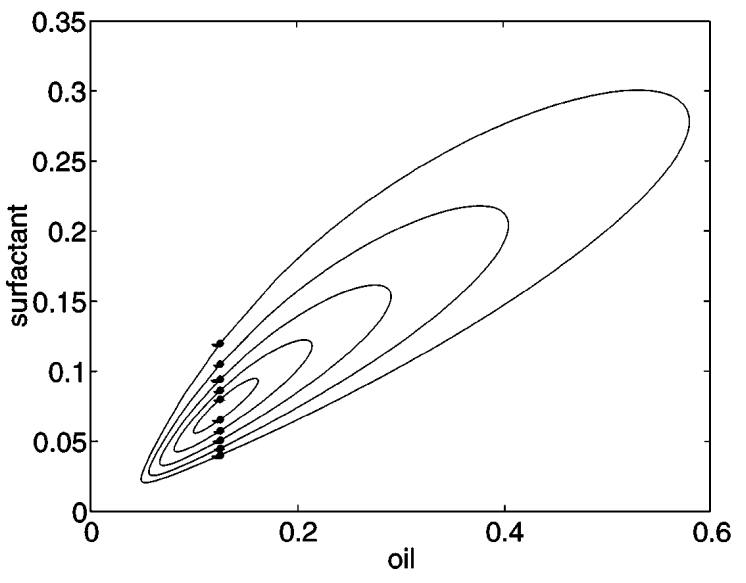

FIG. 1. Spinodal curves: each expanding loop has two critical points at $\phi=\phi_{c} \simeq 1 / 8$. The small loops are symmetrical, and as they grow the lower $\phi$ side of the loop sharpens. The axis of the loops is at $c_{0} r=1 / 2$ in accord with experiment $[1,9]$.

which $c_{0}$ vanishes, both from above and below the length scale, $c_{0}^{-1}$, increases as $c_{0}^{-1} \sim|T-\bar{T}|^{-1}$. Thus, the junctions become larger and, due to the renormalization of the bending modulus, softer, so that the entropy induced attraction between the junctions overcomes the curvature energy cost of their creation. Consequently, there are two double-critical temperatures, $T_{d}=T_{-}, T_{+}$, where the attraction reaches a critical value and the closed loops appear. The water side loop appears at $T_{-}$. As the temperature is increased the double-critical point splits into two separate critical points with radii below and above $c_{0}^{-1} / 2$, both at the same volume fraction $\phi=\phi_{c} \simeq 1 / 8$. The oil side loop exhibits an analogous behavior with an opposite temperature dependence: they shrink with increasing temperature and disappear at $T_{+}$. The difference between the critical radii, $r_{c}$, and the axis of the loop, $c_{0} r=1 / 2$, which is proportional to the width of the closed loops in the isothermal cuts, scales like [23]

$$
c_{0}\left|r_{c}-r_{0}\right| \simeq\left[\epsilon_{2} \frac{\kappa_{0}}{\bar{T}}\left(\frac{T_{+}-T_{-}}{\bar{T}}\right)\right]^{1 / 2}\left|\frac{T-T_{d}}{\bar{T}}\right|^{1 / 2},
$$

where $T_{d}=T_{-}$on the water-rich side and $T_{d}=T_{+}$on the oil-rich side. This temperature dependence is plotted in Fig. 2 along with the experimental data. The oil-rich side data is for a ternary system whose surfactant is $C_{12} E_{5}$ and the water-rich side of a system with $C_{8} E_{4}$. These ternary systems have almost equal values of $\kappa_{0}\left(T_{+}-T_{-}\right)$ [9]. As predicted from Eq. (4), both curves exhibit the square root temperature dependence with almost equal prefactors demonstrating the water-oil symmetry. This indicates that the phase-separation mechanism is identical for both the water and oil systems and is very unlikely to be related to specific attractive interactions.

This branched ME picture also explains the creation of the three-phase triangle as a result of the intersection of

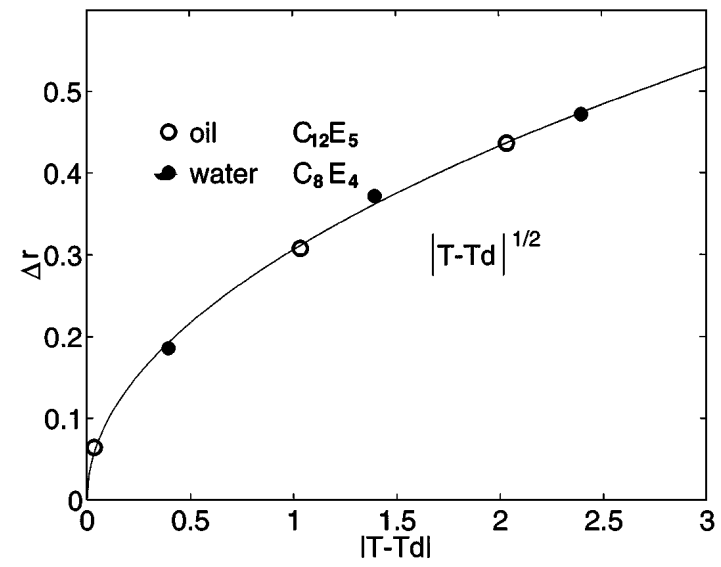

FIG. 2. Expansion of the closed loop: the experimental data from Ref. [7] for the water-rich side of $C_{8} E_{4}$ system and the oil-rich side of $C_{12} E_{5}$ is plotted along the scaling result $\Delta r \sim\left|T-T_{d}\right|^{1 / 2}$. The prefactors for the oil-rich and the water-rich sides are almost the same indicating the water-oil symmetry.

the EF, $c_{0} r=1$, with the closed loop, as seen experimentally [1]. As shown above, when the temperature approaches $\bar{T}$ the closed loop expands. The ratio between the angles of the axis of the loop $c_{0} r=1 / 2$ and the EF $c_{0} r=1$ remains constant. Eventually, the expanding loop intersects the EF and forms a three-phase triangle. There are two intersection temperatures at which the three-phase body appears, $T_{l}<\bar{T}$ for the water side and $T_{u}>\bar{T}$ for the oil side. When the loop further expands its contact point with the EF splits into two phases with volume fractions above and below $\phi_{c}$ which coexist with the excess phase rejected by the globules that have already reached their optimal size. As the temperature approaches $\bar{T}$, the volume fraction of the dense network, usually termed "the middle phase," increases and it becomes an asymmetric sponge phase. At temperatures lower than $\bar{T}$ the asymmetric sponge is composed of connected oil tubes. As the temperature increases the middle phase becomes a symmetric sponge exactly at $\bar{T}$, and after a further temperature increase the asymmetric sponge becomes a net of water tubes.

The interfacial, material-independent nature of our model provides universal scaling laws which are in agreement with a data collapse of several ternary systems onto a single curve when they are described by appropriate reduced variables [22]. Employing the scaling laws which approximate the expansion of the loop (4) and the curvature energy of the junction (3) one derives a universal scaling for the composition of the middle phase, $\phi_{\mu}$, which shows a sigmoidal temperature dependence [23],

$$
\begin{aligned}
\bar{\phi}=\operatorname{sgn}(t)\{[ & \sqrt{(q+1)^{2} / 4-q|t|} \\
& \left.-(q-1) / 2]^{1 / 2}-1\right\},
\end{aligned}
$$




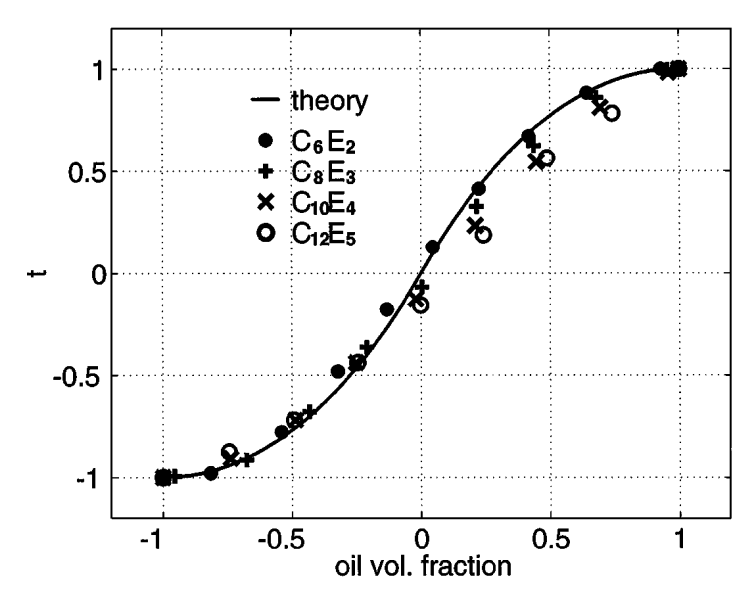

FIG. 3. The middle phase trajectory: the experimental data of several $C_{i} E_{j}$ systems from Ref. [22] collapse onto the theoretical scaling result when plotted in the normalized variables $t=(T-\bar{T}) /\left(\bar{T}-T_{l}\right)=(T-\bar{T}) /\left(T_{u}-\bar{T}\right)$ and $\bar{\phi}=$ $\left(\phi_{\mu}-1 / 2\right) /\left(1 / 2-\phi_{c}\right)$.

where $q \simeq 5$ is a universal constant and the reduced variables are $t=(T-\bar{T}) /\left(\bar{T}-T_{l}\right)=(T-\bar{T}) /\left(T_{u}-\bar{T}\right)$ and $\bar{\phi}=\left(\phi_{\mu}-1 / 2\right) /\left(1 / 2-\phi_{c}\right)$. The middle-phase trajectory (5) exhibits the same water-oil symmetry observed in the closed loops (4). As can be seen from Fig. 3 the theoretical universal curve (5) is in good agreement with the data collapse obtained for four different $C_{i} E_{j}$ surfactants taken from [22]. It is important to note that although the network picture is not valid very close to $\bar{T}$ the scaling for the middle-phase composition passes smoothly through the symmetric sponge phase; thus, indicating that branched ME model is applicable for most of the phase space.

The authors thank B. Widom and P. Pincus for the useful discussions. They are grateful to the Israel Science Foundation and to the Israel Ministry of Science under the joint Israel-French cooperation program.

[1] For a review of experiments R. Strey, Ber. Bunsen-Ges. Phys. Chem. 100, 182 (1996).

[2] P. Kilpatrick et al., J. Phys. Chem. 90, 5292 (1986); M. Kahlweit, R. Strey, and G. Busse, Phys. Rev. E 47, 4197 (1993).

[3] S. A. Safran and L. A. Turkevich, Phys. Rev. Lett. 50, 1930 (1983); M. S. Leaver and U. Olsson, Langmuir 10, 3449 (1994).

[4] In ionic systems the existence of extra degree of freedom, the concentration of the salt, complicates the phase diagram. Nevertheless, when the ratio water/oil is kept constant and the control parameter is the salinity (analogous to the temperature in nonionic systems) it exhibits a similar progression of the phase behavior, including the appearance of closed loops and three-phase body.

[5] M. S. Laver, U. Olsson, H. Wennerström, and R. Strey, J. Phys II (France) 4, 515 (1994).
[6] O. Glatter, R. Strey, K.-V. Schubert, and E. W. Kaler, Ber. Bunsen-Ges. Phys. Chem. 100, 323 (1996).

[7] R. Strey, Colloid Polymer Sci. 272, 1005 (1994).

[8] I. S. Barnes, S. T. Hyde, B. W. Ninham, P. J. Derian, M. Drifford, and T. N. Zemb, J. Phys. Chem. 92, 2286 (1988).

[9] R. Strey (unpublished).

[10] G. Gompper and M. Schick, Self-Assembling Amphiphilic Systems (Academic, New York, 1994).

[11] S. T. Milner, S. A. Safran, D. Andelman, M. E. Cates, and D. Roux, J. Phys. (Paris) 49, 1065 (1988); P. Pieruschka and S. A. Safran, Europhys. Lett. 31, 207 (1995).

[12] S. A. Safran, L. A. Turkevich, and P. Pincus, J. Phys. (Paris) Lett. 45, L-69 (1984).

[13] R. Menes, S. A. Safran, and R. Strey, Phys. Rev. Lett. 74, 3399 (1995).

[14] Systems with negative saddle-splay modulus, $\bar{\kappa}<0$ prefer minimal number of arms, $z=3$. This is in accordance with the observation of " $Y$-like" structures by Strey et al. [7] which suggests 3-fold junctions.

[15] T. J. Drye and M.E. Cates, J. Chem. Phys. 96, 1367 (1992). The statistical mechanics of the network are treated by a method similar to what they use for micellar networks. The entropy penalty is $-(z \rho \ln z \rho-\rho \ln \rho) \simeq$ $-(z-1) \rho \ln \rho$. It vanishes in the limit $z=1$ where Eq. (1) reproduces the result for wormlike micelles. The different underlying microscopic properties are taken into account in the dependence of the junction bending energy, $\epsilon$, on the swelling radius, $r$, leading to a phase separation unique for ME networks.

[16] This assumption fails for thick cylinders in the vicinity of the EF, where the junction energy, $\epsilon$, is comparable with the end-cap energy and the network is unstable to the formation of nearly spherical drops.

[17] W. Wintz, H.-G. Dobreiner, and U. Seifert, Europhys. Lett. 33, 403 (1996).

[18] This approximates the bending energy, $\kappa \int\left(H-c_{0}\right)^{2} d A$, of the junction. The Gaussian curvature contribution to the junction bending energy is $-4 \pi \bar{\kappa}(z / 2-1)=-2 \pi \bar{\kappa}[\mathrm{Z}$. Wang and S. A. Safran, Europhys. Lett. 11, 425 (1990)]. This contribution does not depend on the cylinder radius and therefore affects only the values of the coefficients, $\epsilon_{0}$ and $\epsilon_{2}$, with no qualitative change in the phase behavior.

[19] D. Roux and C. Coulon, J. Phys. (Paris) 47, 1257 (1986); J. V. Selinger and R. F. Bruinsma, Phys. Rev. A 43, 2910 (1991). The energy per unit length associated with the entropy of a cylinder fluctuating inside a tube of radius $d$ scales like $\left(\kappa r d^{2}\right)^{-1 / 3}$. In our model conservation implies $\phi \sim(d / r)^{2}$. The cylinder length per unit volume scales like $\phi / r^{2}$. Employing these scalings we find the $\phi^{4 / 3}$ dependence of $F_{H}$.

[20] In dense networks, when the junctions are close, they strongly interact via the elastic deformations imposed by their dense packing. However, this extra repulsion is minor in the vicinity of the critical point.

[21] For example, L. Peliti and S. Leibler, Phys. Rev. Lett. 54, 1690 (1985).

[22] T. Sottmann and R. Strey, J. Phys. Condens. Matter 8, A39 (1996).

[23] T. Tlusty, S. A. Safran, R. Menes, and R. Strey (to be published). 